La

Révolution

française

\section{La Révolution française}

Cahiers de l'Institut d'histoire de la Révolution française

$17 \mid 2020$

Gouverner par la loi

\title{
Anne DE MATHAN (dir.), Mémoires de la Révolution française, Rennes, Presses universitaires de Rennes, 2019, 391 p.
}

Baptiste Roger-Lacan

\section{OpenEdition}

Journals

Édition électronique

URL : http://journals.openedition.org//rf/3647

DOI : $10.4000 /$ Irf.3647

ISSN : 2105-2557

Éditeur

IHMC - Institut d'histoire moderne et contemporaine (UMR 8066)

Référence électronique

Baptiste Roger-Lacan, « Anne de mathan (dir.), Mémoires de la Révolution française, Rennes, Presses universitaires de Rennes, 2019, 391 p. », La Révolution française [En ligne], 17 | 2020, mis en ligne le 24 février 2020, consulté le 20 avril 2020. URL : http://journals.openedition.org//rf/3647 ; DOI : https:// doi.org/10.4000/Irf.3647

Ce document a été généré automatiquement le 20 avril 2020.

(c) La Révolution française 


\title{
Anne DE MATHAN (dir.), Mémoires de la Révolution française, Rennes, Presses universitaires de Rennes, 2019, 391 p.
}

\author{
Baptiste Roger-Lacan
}

1 «C'est quelque chose de grand que la puissance de la mémoire. Une sorte d'horreur me glace, ô mon Dieu, quand je pénètre dans cette multiplicité profonde, infinie ${ }^{1}$ ! » Les deux premières phrases de la citation de Saint Augustin qui ouvre l'introduction d'Anne de Mathan à l'ouvrage collectif qu'elle a dirigé, Mémoires de la Révolution française, ont quelque chose de profondément et justement programmatique quant à l'ampleur de la tâche qu'elle a confiée à l'ensemble des contributeurs de ce livre important: rendre compte par la multiplication des perspectives du problème historique que pose la mémoire de la Révolution française. Si la diversité des approches - avec l'introduction et la conclusion, l'ouvrage compte trente-deux articles écrits par trente-et-un chercheuses et chercheurs venus du monde entier - est l'une des grandes richesses de ce livre, elle complique la tâche du commentateur s'il veut rendre compte aussi bien des grands axes qui le structurent que des nombreuses pistes qui sont ouvertes par chacun des articles.

2 Anne de Mathan, maître de conférences à l'Université de Bretagne Occidentale, dirige cet ouvrage qui s'inscrit dans le sillon de ses travaux précédents sur la mémoire en Révolution ${ }^{2}$. Dès son introduction, "La pique, la plume et le cœur. Des liens entre historiographie, mémoire et politique», elle explicite le fait que la mémoire qui sera discutée dans ce livre est autant celle qui fait depuis longtemps l'objet d'une investigation scientifique et philosophique que celle qui compose avec l'histoire les deux faces d'un même problème, enregistrer et transmettre ce qui a eu lieu. Bien qu'appartenant à des ordres différents, l'histoire et la mémoire «entretiennent toutefois un certain commerce, ne serait-ce que parce qu'elles visent un même objet, à savoir ce qui est révolu3 ${ }^{3}$ » De plus, bien souvent, l'une est appelée à devenir l'autre et 
inversement, Anne de Mathan prenant ainsi l'exemple des cours d'histoire appelés à se fixer dans la mémoire des étudiants qui l'entendent et, dans l'autre sens, des mémoires spécifiques, comme celle de la Vendée ou de certains personnages de la Révolution, sont appelées à devenir des objets historiographiques. Ce texte est aussi l'occasion de faire une généalogie des recherches sur la mémoire, qu'Anne de Mathan situe au moment de l'émergence de la modernité littéraire sous les auspices de Bergson, Proust et Freud.

3 Ces premières incursions dans la mémoire furent ensuite formalisées dans le champ des sciences sociales par Maurice Halbwachs, l'une des figures tutélaires de ce livre, dont Les cadres sociaux de la mémoire (1925) ont fixé le concept de « mémoire collective » et de "mémoire sociale». Anne de Mathan souligne également le rôle-pionnier de March Bloch qui, dans Les rois thaumaturges (1924), s'intéresse longuement au problème de la mémoire. Ce n'est cependant pas avant les années 1950 et 1960 que la mémoire, par le biais de l'entreprise collective de l'oral history dans le monde anglo-saxon et celle, individuelle, de Philippe Joutard sur la mémoire camisarde dans les Cévennes, que la mémoire devient un objet d'histoire à part entière. C'est le début d'une "révolution mémorielle» - Anne de Mathan reprend ici une expression de Patrick-Michel Noël -, qui passe par de nombreux recueils de témoignages, faits par des historiens mais aussi par des généalogistes amateurs ou par des institutions publiques ou privées. Cette inflation mémorielle a plusieurs conséquences: elle contribue à l'émergence de nouveaux discours identitaires ou communautaires ancrés dans le mémoriel; elle peut aboutir à des guerres de mémoire; elle voit l'État s'engager sous une forme nouvelle, celle des politiques mémorielles, qui, pour Anne de Mathan, « révèlent l'impuissance de l'action politique dans le présent et la difficulté de la projection dans l'avenir ${ }^{4}$ », tout en démontrant une volonté de sauver un roman national de plus en plus déconnecté des travaux historiques.

4 Face à un discours mémoriel qui concurrence l'histoire " comme discours d'autorité sur le passé5 ", Anne de Mathan rappelle que les historiens s'emparent de l'objet «mémoire » à la fin des années 1970: en 1978, François Bédarida fonde l'Institut d'Histoire du Temps Présent ; parallèlement l'histoire de la mémoire devient un nouvel enjeu de la recherche dont Les lieux de mémoire ${ }^{6}$ sont une première manifestation de cette réflexion d'historien sur la mémoire collective. Et bien qu'Anne de Mathan souligne les défauts des Lieux de mémoire, Mémoires de la Révolution française est aussi une manière pour les historiens de porter un discours scientifique sur une passion individuelle et collective, la mémoire, qui vient souvent concurrencer leur activité. Du reste, comme Les lieux de mémoire, le présent ouvrage est issu des travaux d'un séminaire, à l'université de Bretagne occidentale entre 2014 et 2015 pour le second. À raison, Anne de Mathan souligne que la grande explosion mémorielle suscitée par la Révolution, qui prend souvent la forme d'une prolongation victimaire du discours contre-révolutionnaire, mérite d'autant plus qu'on la questionne que l'outillage conceptuel à disposition des chercheurs a considérablement évolué depuis le bicentenaire de la Révolution?

5 L'armature conceptuelle de ce livre est influencée par ce constat et c'est ainsi que la première partie du livre, "La mémoire sur l'établi des sciences sociales", que l'introduction d'Anne de Mathan anticipe à certains égards, est consacrée à un tour d'horizon des enjeux épistémologiques de la mémoire en sciences sociales et, plus spécifiquement, de la mémoire de la Révolution française. La deuxième partie, «Jeux de 
miroirs : le pluriel des historiographies de la Révolution française ", perd un peu de vue la stricte question mémorielle - on y reviendra - pour envisager les historiographies de la Révolution dans une perspective internationale. Enfin, la troisième partie, intitulée "Des histoire de mémoire de la Révolution française", se divise en deux échelles, individuelle et collective, et permet à quinze historiennes et historiens de présenter des exemples de mémoire de la Révolution depuis 1789 jusqu'à l'époque contemporaine. Avec ses trente-et-une contributions, la proposition de ces Mémoires de la Révolution française est donc singulièrement riche, quoique tenue par ces trois - ou quatre si l'on considère que la dernière partie se divise en deux - grands axes de recherche et de réflexion.

6 Le premier mouvement de ce livre, qui s'intéresse aux travaux contemporains sur la mémoire, commence là où elle s'arrête dans sa généalogie de la mémoire. Aussi diverses par leurs objets que par les approches retenues, toutes ses contributions ont en commun de vouloir dépasser une opposition, jugée stérile, entre histoire et mémoire.

7 Les deux premières - «Ce que les sciences sociales nous disent de la mémoire » de Michèle Baussant et «La mémoire comme objet des sciences sociales » de Marie-Claire Lavabre - sont indissociables dans la mesure où elles apportent un éclairage synoptique aux chapitres suivants. Dans sa contribution, Michèle Baussant donne à voir avec virtuosité les nombreuses formes de rapport au passé que le concept de mémoire peut recouvrir dans les différents champs des sciences sociales. En partant notamment des œuvres de Michel de Certeau et de Paul Ricœur, et au prisme des contributions du livre, elle invite à dépasser la dénonciation des abus de la mémoire qui découle d'une conception trop unanimement politique de la mémoire. Par un travail de définition et délimitation précis des différentes formes d'approche du passé, elle propose ainsi de dépasser l'opposition entre histoire et mémoire. Dans la contribution de Marie-Claire Lavabre, ce n'est pas tant la mémoire que la « mémoire collective » d'abord étudiée par Maurice Halbwachs qui est centrale. Elle s'engage dans une critique de ce concept presque séculaire au prisme des différents champs qui ont depuis été ouverts sur la question de la mémoire. En passant notamment par les trois notions clefs que sont les "lieux de mémoire ", identifiés par Pierre Nora, le "travail de mémoire ", associé à Paul Ricœur, et les « cadres de la mémoire ", au cœur du travail d'Halbwachs lui-même, Marie-Claire Lavabre s'attaque bien aux malentendus et aux contresens qu'ont pu susciter des notions parfois surinvesties par les chercheurs en sciences sociales. L'explicitation des définitions que donnent l'histoire, la sociologie et même la psychanalyse de ce concept de "mémoire collective» montre que celles-ci se complètent bien plus qu'elles ne s'opposent et l'identifient comme un rapport au passé parmi d'autre. Marie-Claire Lavabre suggère alors, par un retour aux intuitions de Halbwachs, que la mémoire collective permet de désigner les circulations entre ces trois formes de rapport au passé, permettant une sociologie du souvenir.

Dans « Travail de mémoire, devoir de mémoire, devoir d'histoire, travail de l'histoire ", le philosophe et politiste Johann Michel, spécialiste de Paul Ricœur, part de ce titre en chiasme pour mener une réflexion qui met en miroir les concepts de travail et devoir 
de mémoire, très utilisés aussi bien dans le champ des sciences sociales que par le grand public, et ceux moins connus, mais que Michel se propose de réévaluer, de devoir et travail de l'histoire. Il entreprend d'abord une généalogie des deux concepts mémoriels en montrant notamment que, loin d'avoir cherché à opposer travail et devoir de mémoire (ou à employer le premier pour remplacer le second), Paul Ricœur a surtout cherché à les articuler de façon dialectique, la simple contrainte du devoir n'étant pas suffisante. Cela précède une réflexion sur les propriétés du devoir et du travail d'histoire. L'un comme l'autre mettent en jeu le rapport phénoménologique de l'historien au passé, médiatisé par le problème de la "trace », et, par conséquent, le problème de son objectivité supposée. Bien plus, le devoir d'histoire est ainsi conçu comme un préalable, une condition nécessaire, au travail *de l'histoire, en cela différent du devoir et du travail de mémoire, qui peuvent parfois se « contrarier ${ }^{8}$ ».

9 À la suite de cette contribution, celle de Philippe Joutard, pionnier de l'historiographie de la mémoire, propose un texte au titre programmatique : « Histoire et mémoire. Deux approches du passé souvent en conflit mais qui doivent s'articuler». Son premier mouvement est de rappeler que mémoire et histoire sont aujourd'hui deux concepts trop souvent confondus, qui semblent être "identiques et interchangeables ${ }^{9}$ ", alors que « tout les oppose ${ }^{10} »$. Son raisonnement consiste à rappeler les fondements de cet antagonisme: sélectivité de la mémoire contre ambition exhaustive de l'histoire; dépendance sensible de la première contre préférence de la seconde pour l'écrit; écrasement du passé sur le présent dans le temps mémoriel lorsque le temps historique cherche à rétablir "la distance ${ }^{11}$ ». Cet inventaire de contraires explique en partie pourquoi le «conflit s'est aggravé avec la poussée mémorielle des années $1970^{12}$ »: l'histoire de la Résistance fut un vrai point d'achoppement, qui vit témoins et historiens s'affronter, parfois avec violence, sur le récit de ce passé. Pour autant, l'exposé de cette opposition invite à un dépassement dont Philippe Joutard, riche de sa longue étude des mémoires, notamment cévenoles, expose les conditions : plutôt que de s'attaquer aux mémoires institutionnelles ou nationales, dont l'ambition entre nécessairement en conflit avec les règles de l'historien, celui-ci ne devrait-il pas plutôt s'intéresser aux "mémoires modestes", des traces onomastiques ou folkloriques, rarement étudiées alors qu'elles portent un discours sur le passé. Cette dernière proposition est particulièrement stimulante et elle ouvre de vrais possibles aux historiens de la Révolution et de la Contre-révolution.

10 Si Philippe Joutard conclut sa contribution par la question de l'étude du folklore comme l'une des portes d'entrée potentielles dans les mémoires «modestes» dont il voulait s'emparer, Laurent Le Gall, quant à lui, ouvre justement la sienne, "Mémoire et tradition au risque de la confusion ", par une réflexion sur les compilations folkloristes de la fin du $\mathrm{XIX}^{\mathrm{e}}$ siècle, et plus généralement sur les entreprises de collecte de la mémoire avant que ce dernier concept ne commence à s'imposer dans le champ historique. Cela lui permet de montrer comment la catégorie historiographique «mémoire » s'est imposée à partir des années 1970, permettant notamment de mettre un mot sur un impensé de nombreux ouvrages d'histoire politique depuis le Tableau politique de la France de l'Ouest d'André Siegfried ${ }^{13}$. S'il ne conteste pas du tout l'importance de ce nouveau paradigme mémoriel, et son impact dans la construction d'identités politiques situées géographiquement en citant par exemple les études fondatrices de Jean-Clément Martin dans les années $1980^{14}$, il avertit aussi contre le risque d'une confusion entre mémoire et tradition, la première servant simplement à constater, sans la critiquer, l'existence de la seconde. Bien plus, en postulant l'existence 
de permanences politiques déconnectées de leurs contextes historiques, il montre comment l'usage indifférent de la mémoire peut ouvrir la voie à une forme de traditionalisme épistémologique au raisonnement parfaitement anhistorique et dont le seul but serait de constater l'existence de traditions politiques, artificiellement fabriquées dans des textes postulant leur existence. Sa contribution se conclut par un retour à Maurice Halbwachs, médiatisé par un commentaire de Raoul Girardet, qui pose quelques pistes pour éviter ce cercle vicieux.

11 En creux, Laurent Le Gall pose la question cruciale du rôle de l'historien qui s'occupe de mémoire : dans quelle mesure son activité en vient-elle à modifier, volontairement ou non, l'objet sur lequel il travaille? L'ethnologue Sylvie Sagnes, dans "De passeur à décrypteur de mémoire ... et retour ", offre de nombreux éléments de réponse inspirés par le parcours de plusieurs ethnologues dont les travaux anciens ont été réappropriés par les membres actuels des communautés sur lesquelles ils avaient travaillé, avec cet effet «de convertir l'ethnographie d'hier en archives d'aujourd'hui et son auteur en témoin ${ }^{15}$ ». Elle va néanmoins plus loin en montrant, notamment à travers la question $\mathrm{du}$ patrimoine culturel immatériel, que cette double étiquette de chercheur et de passeur de mémoires finit par se retrouver aussi dans la recherche contemporaine, l'ethnologue étant de plus en plus considéré comme le "spécialiste tout désigné de l'immatérialité16 ». Engagés dans leur processus de collecte, elle montre la difficulté des ethnologues à trouver de la distance avec leur objet alors que la question de la mémoire les entoure. À cet égard, ce détour par l'ethnologie pose de vraies questions aux historiens, dont la pratique, en plus d'être concurrencée par les discours mémoriels, risque parfois de ne devenir qu'un conservatoire de traditions et de mémoire, dont l'ambition scientifique serait perdue ou incomprise par son public.

"Quand l'histoire bute sur les mémoires, le cas de la Révolution française ", dernière contribution de cette partie, voit Jean-Clément Martin partir d'un essai publié il y a quelques années ${ }^{17}$ et dans lequel il comparait le lien entre histoire et mémoire à celui du ruban de Moebius, « insécable, permettant le passage d'une extrémité à l'autre sans coupure $^{18}$ ». En refusant une lecture de la Révolution qui en ferait un «bloc» ou une " rupture » nette avec le passé, il essaye de comprendre comment les révolutionnaires, à différents moments de la Révolution (plutôt avant 1794 qu'après), ont voulu jouer l'histoire contre la mémoire de l'Ancien régime, celle-ci devant être remplacée par un ensemble de rites et de pratiques mémorielles nouvelles et d'autant plus souvent concurrentes qu'elles permettaient aux différents groupes qui s'affrontaient dans le champ du pouvoir de légitimer leurs actions autant que de discréditer celles de leurs rivaux. Jean-Clément Martin montre que, loin d'être une invention de 1789, ce programme de refondation mémorielle trouve sa source dans les nombreux travaux qui, partout en Europe et dans les colonies, cherchent à moderniser et rationnaliser l'action de l'État. Parallèlement, les mémoires politiques prérévolutionnaires eurent beaucoup d'importance dans la détermination politique des groupes d'acteurs qui s'affrontèrent au centre ou dans les périphéries de la France. Cette première sédimentation historico-mémorielle contemporaine des évènements révolutionnaires jette les fondements de ce qui est amené à devenir un ensemble de "mémoires antagonistes» dont l'article retrace synthétiquement les évolutions depuis 1789. Cependant, loin d'être perçue de manière exclusivement négative, cette guerre civile mémorielle contribue pour Jean-Clément Martin à la vitalité des identités, nationale et locales, en France. À ce conflit mémoriel intérieur s'ajoute la diversité des mémoires nationales de la Révolution que celle-ci a suscitées dans le reste du monde: du 
Royaume-Uni à l'Équateur, cet article balaie certaines de ces mémoires (favorables ou hostiles). La conséquence la plus problématique pour l'historien d'un conflit mémoriel, sans cesse réagencé au prisme de nouvelles lignes de fracture politique ou culturelle, est qu'il empêche, selon Jean-Clément Martin, "la constitution d'une communauté historique authentique » sur la Révolution, dans la mesure où des groupes d'historien se feraient les défenseurs de mémoires concurrentes. Pour dépasser ce blocage, il suggère de commencer à considérer la Révolution aussi comme une guerre civile ce qui "permettrait de "désidéologiser" la compréhension des massacres et des tueries ${ }^{19}$ ". C'est, pour lui, seulement au prix de ce deuil d'une lecture idéologique et statique de la Révolution qu'il sera possible pour les historiens de reprendre le contrôle sur l'histoire de la Révolution.

En ne limitant pas sa contribution aux seules mémoires françaises de la Révolution (et à leur impact sur des historiographies concurrentes) Jean-Clément Martin prépare la deuxième partie de cet ouvrage, « Jeux de miroirs : le pluriel des historiographies de la Révolution française ", dans laquelle huit contributions entendent rendre compte de la diversité des historiographies de la Révolution et de leurs liens avec les mémoires collectives ou individuelles qui les précèdent et, souvent, les orientent. Des cas gallois, français, britannique, soviétique ou encore italien sont ainsi étudiés, donnant un aperçu de la grande diversité des interprétations suscitées par l'objet «Révolution française » depuis deux siècles.

Dans cette partie, la contribution d'Annie Jourdan, « Au-delà des frontières. Dialogues de sourds et miroirs déformants ", tranche avec celles qui la suivent. De fait, loin de se limiter à l'étude d'un cas national, elle entreprend de donner un aperçu global des écritures immédiates de la Révolution française qui en sont données au-delà des frontières à partir de 1789. Immédiatement, en effet, deux lectures s'affrontent: celle des défenseurs de la Révolution, qui espèrent que son modèle se propagera dans leur pays ; et celle de ses opposants, qui dénoncent une entreprise ancrée dans l'abstraction et la négation de la réalité qui ne peut que virer au désastre, une tradition dont Edmund Burke est l'un des plus éminents représentants à partir de 1790. À mesure que le processus révolutionnaire pèse de plus en plus sur l'équilibre diplomatique européen, ces récits originels sont souvent modifiés dans le sens d'interprétations plus hostiles. Très vite en somme, «la diabolisation de la révolution est en fait partie intégrante des mythes généalogiques de l'Occident. ${ }^{20} »$. De fait, les contributions de la deuxième partie de l'ouvrage - qui traitent, à l'exception de la dernière, d'historiographies plutôt favorables à la Révolution - permettent de mesurer leur grande variété, mais aussi le lien fort qui existe entre ces récits historiques et leurs contextes d'écriture. C'est que, pour Annie Jourdan, une histoire de la Révolution nous apprend souvent moins sur celle-ci que sur « la face cachée de [l']histoire nationale ${ }^{21} »$ de ceux qui les écrivent.

Ainsi, le cas de la réception de l'œuvre de Richard Price (1723-1791), un Gallois ami de la Révolution, témoigne de la pluralité des mémoires de la Révolution depuis 1789 en France, au Pays de Galles ou dans le reste du monde anglo-saxon, autant que de la manière dont ces mémoires sont directement tributaires des contextes politiques et culturels différenciés dans lesquels elles s'expriment. Rémy Duthille, dans «Richard 
Price (1723-1791). Gloire, éclipse et redécouvertes d'un Gallois ami de la Révolution française ", montre ainsi comment Price, promoteur de la Révolution dès le mois de novembre 1789, connut une heure de gloire précoce en France, notamment à cause des attaques que Burke déploya contre lui dans ses Réflexions sur la Révolution de France. Sa mort en 1791, autant que l'effacement progressif du cosmopolitisme, qu'il était venu à incarner avec d'autres soutiens étrangers de la Révolution, devant les urgences de la guerre intérieure et extérieure, font que Price s'effaça en France alors qu'il était attaqué en Angleterre. En quelque sorte précurseur de Paine et Anarchasis Cloots, il ne connaît pourtant pas leur célébrité et, pendant longtemps, il est marginalisé dans l'historiographie française et, surtout, anglo-saxonne. Ainsi, l'historiographie du radicalisme anglais, et notamment E. P. Thompson, démontre qu'il est trop modéré pour les artisans jacobins de Sheffield ou York. C'est à partir des travaux de Godechot et Palmer, qui citent certains des pamphlets de Price, que celui-ci sort progressivement de l'obscurité, son cas faisant l'objet de débat, notamment sur le problème de son appartenance aux très discutées Lumières radicales. Parallèlement émerge au Pays de Galles une mémoire nationale qui en fait l'un des penseurs les plus originaux fournis par la principauté. Dans ce nouveau paradigme mémoriel ce sont moins ses analyses des révolutions de 1688 et 1789 qui sont retenues que sa «nationalité » littéraire. À travers le cas de Richard Price, on mesure ainsi comment une figure marginalisée dans les historiographies française et anglaise de la Révolution, pourtant souvent concurrentes, peut faire l'objet d'un réinvestissement mémoriel dans lequel la Révolution apparaît à son tour comme marginale.

16 Si Richard Price n'est pas une figure centrale des mémoires ou des historiographies de la Révolution, Robespierre est peut-être la figure la plus associée à celle-ci. Alors que celui-ci devient un "enjeu historiographique ${ }^{22}$ » autant que politique depuis une dizaine d'années, Yannick Bosc, dans «Robespierre, la gauche et la fabrique du négatif ", s'emploie à démêler les différentes traditions qui, à gauche, ont contribué à en construire une image négative. Il distingue trois registres qui fabriquent ce négatif : les récits franchement hostiles qui, des républicains aux blanquistes, font de Robespierre une antithèse de la Révolution qu'il aurait détruite; les récits contreproductifs qui, à force d'hagiographie et d'orthodoxie stalinienne, ont démonétisé politiquement le personnage; la muséification qui voit Robespierre assigné à une Révolution bourgeoise déjà datée à la fin du XIX siècle. Malgré le brouillage de la figure de Robespierre qu'ont provoqué ces discours historiques contradictoires venus de la gauche, Yannick Bosc constate néanmoins qu'il demeure une figure vivante, comme le démontrent les nombreux débats - aussi bien dans le champ médiatique que dans celui de l'histoire - qu'il suscite encore, comme l'illustrait récemment la sortie de l'opus du jeu vidéo Assassin's Creed consacré à la Révolution ${ }^{23}$.

17 Après deux réflexions autour de "figures ", la contribution d'Antonino de Francesco, «1789 et l'historiographie italienne. Une reconfiguration nationale de la Révolution française ", cherche au contraire à rendre compte des évolutions de l'historiographie italienne de la Révolution depuis le début du XIx siècle jusqu'à aujourd'hui. Deux tensions sont à l'œuvre : la première est proprement italienne, dans le sens où la lecture libérale et la lecture nationaliste de la Révolution qui évoluent concomitamment du XIX siècle jusqu'en 1945 sont caractérisées par un discours résolument hostile à la Révolution; inversement pourtant, les historiens italiens ne cessent de dialoguer avec leurs collègues français, jusque dans leurs silences qui 
témoignent souvent de tentatives de s'approprier politiquement tel ou tel moment de la Révolution française. Le contexte de l'Italie post-fasciste permet de soulager le débat transalpin de certaines de ses tensions, mais il impose aussi de nouvelles grilles de lecture qui voient notamment les historiens italiens adopter, dans le sillage partiel de Guglielmo Ferrero, une lecture positive des années 1789 à 1792, balancée par une approche très critique de la période du gouvernement révolutionnaire, prémisse d'une dictature implicitement associée au fascisme. Cette dichotomie entre l'avant et l'après 1793, selon Antonino de Francesco, conserve une forme d'influence jusqu'à aujourd'hui et contribue au manque de renouvellement des études révolutionnaires en Italie.

Comme son titre l'indique, la contribution d'Alexandre Tchoudinov, "Les recherches sur la Révolution en URSS. Principaux jalons", prend la forme d'une introduction synthétique et structurée de l'histoire de l'historiographie révolutionnaire soviétique. Pour cette raison, ce chapitre est difficile à résumer bien qu'il offre au néophyte un exposé très clair de l'histoire de l'école soviétique de la Révolution, de son apparition aux lendemains de la Révolution de 1917 jusqu'à sa disparition très rapide après la disparition de l'Union soviétique. On peut néanmoins regretter que ne soit pas plus discutée la notion d'«école", tout particulièrement dans un contexte où l'État surinvestit le champ universitaire.

Dans « En finir avec Sartre, en finir avec la Révolution française ? Les années 1960 face à un objet surinvesti », Sophie Wahnich analyse, d'une part, comment Jean-Paul Sartre s'est emparé, pour le modeler à la mesure de sa pensée, de l'objet «Révolution française » et, d'autre part, comment les intellectuels dits structuralistes ont cherché à se débarrasser de la Révolution dans leurs attaques contre Sartre. Elle montre d'abord comment ce dernier utilise l'évènement révolutionnaire pour développer sa philosophie de la liberté individuelle et collective. Alors que cette approche est vivement critiquée par les structuralistes, notamment Lévi-Strauss, Lacan, Foucault et Althusser, ceux-ci vont chercher à refroidir la Révolution. En développant les cas de Lévi-Strauss puis de Foucault, Sophie Wahnich montre comment ceux-ci ont cherché à nier la rupture révolutionnaire avec pour lourde conséquence que l'objet Révolution, " après avoir été installé par l'historiographie marxiste, communiste puis sartrienne au point d'acmé de l'imaginaire politique ", avait été transformée en objet inerte par les opposants à Sartre. La Révolution française, un zombie historiographique ? C'est ce que suggère Sophie Wahnich dans sa conclusion.

Dans sa contribution, «Walter Markov : la "Grande Révolution française" vue de l'Est ", Jean-Numa Ducange entremêle l'itinéraire singulier de Walter Markov, historien allemand spécialiste, entre autres, des Enragés, à celui de l'historiographie estallemande de la Révolution. Markov, « dissident de l'intérieur ${ }^{24}$ » qui n'a jamais voulu quitter la RDA et qui est mort quelques années après la chute du mur de Berlin, en 1993, a contribué à un champ original en Allemagne de l'Est, celui des Enragés, un groupe souvent associé à l'extrême gauche, ce qui démontrait une réelle originalité dans un pays connu pour son respect de l'orthodoxie stalinienne. Au cours de son texte, JeanNuma Ducange cherche à la fois à expliquer l'effacement actuel de la figure de Markov et à comprendre comment celui-ci a cherché à articuler des circulations scientifiques entre les deux Allemagnes ou avec la France à une époque de fortes tensions politiques et diplomatiques. À cet égard, son travail sur Jacques Roux et les Enragés se lit aussi comme une manière de reprendre le fil interrompu par la grande glaciation historiographique de la fin des années 1930 qui suit les grandes purges. Travailler sur 
les Enragés dans un contexte où il fallait justifier ses recherches dans le cadre marxisteléniniste apparaissait sans doute à Markov comme un moyen de promouvoir des pratiques démocratiques populaire. En ce sens, il témoignait peut-être d'un discret agenda politique et, surtout, il réussit à influencer l'historiographie classique des Enragés. Aujourd'hui, la mémoire de Markov, trop associée au régime disparu en 1989, a considérablement faibli. Mais le travail historique de Jean-Numa Ducange, qui restitue l'importance de cet auteur dans la constitution d'une historiographie «par en bas ", participe aussi d'une forme de logique mémorielle en restaurant l'image d'un historien quelque peu oublié. Du reste, une note de bas de page témoigne que le souvenir qu'en avaient certains participants du séminaire d'Anne de Mathan a en partie animé la discussion autour de la présentation de Jean-Numa Ducange.

21 En conclusion de cette deuxième partie, le texte de Paul Chopelin est le seul à traiter d'un groupe marqué par les récits contre-révolutionnaires. « Du Bicentenaire à la Manif pour tous. Les catholiques français et la mémoire de la "persécution révolutionnaire" (années 1980-années 2010) » rend compte des ambivalences et des contradictions de la relation qu'entretiennent l'Église et les catholiques observants avec l'histoire de la Révolution française. Son introduction revient d'abord sur la manière dont l'Église a promu, jusque dans les années 1950, une mémoire hostile à la Révolution, avant de faire silence sur un évènement que la hiérarchie catholique se refuse à qualifier positivement ou négativement. Pour Paul Chopelin, le Bicentenaire vient raviver les contradictions dans lesquelles évoluent les catholiques quant à la Révolution : d'un côté, ils soutiennent très largement la démocratie républicaine; de l'autre, ils veulent conserver le souvenir de leurs victimes. Les commémorations du Bicentenaire entretiennent le malaise tandis que l'Église, comme les autres institutions concernées par celles-ci, campe sur ses positions historiographiques : approbation de la libération de 1789 et dénonciation des " horreurs » qui ont suivi. À la suite de 1989, on assiste plutôt à un retour en force d'une mémoire hostile à la Révolution. Malgré quelques initiatives ponctuelles, comme la réhabilitation de l'abbé Grégoire par Monseigneur Maurice de Germigny, son lointain successeur au diocèse de Blois, l'Église continue de béatifier des religieux tués pendant la Révolution alors qu'une partie des catholiques français réinvestissent un discours contre-révolutionnaire et hostile à l'Université, relayé aussi bien sur internet que dans certains médias destinés au grand public. La Manif pour tous participe ainsi de cette crispation mémorielle catholique alors que de nombreux militants « tradismatiques ${ }^{25}$ ", pour reprendre l'expression du politologue Gaël Brustier, cultivent l'analogie avec des figures chouannes ou vendéennes. Pour expliquer ce retour de conflictualité mémorielle, Paul Chopelin suggère que l'incapacité de la Mission du Bicentenaire à s'emparer des "mémoires divergentes ${ }^{26}$ " de la Révolution a fermé la voie à une réconciliation mémorielle en permettant au contraire la légitimation de contrehistoires de la Révolution reposant sur une lecture contre-révolutionnaire et souvent franchement conspirationniste.

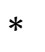

"Des histoires de mémoire de la Révolution française ", la troisième partie de cet ouvrage, se présente comme l'aboutissement de la réflexion entamée dans l'introduction. L'exposition des problèmes épistémologiques soulevés par la mémoire en première partie et, dans un deuxième temps, l'étude de plusieurs contextes 
historiographiques - à différentes époques et dans différents espaces - viennent préparer cette collection d'études historiques sur la mémoire de la Révolution. Cette dernière partie, avec ses quinze contributions, représente près de la moitié de l'ouvrage: après une contribution introductive de Pierre Serna, il a été décidé d'organiser les autres textes en deux groupes qui interrogent ces mémoires de la Révolution à deux échelles, individuelle et collective. Il faut également noter que contrairement à la deuxième partie, qui traitait largement des historiographies nonfrançaises de la Révolution, la troisième est complètement recentrée sur le cadre national.

La contribution introductive, et programmatique, de Pierre Serna, "Régimes de mémoires et mémoires de régimes. Une histoire impossible?", revendique une double filiation historiographique : celle de Sergio Luzzato, dont Mémoire de la Terreur ${ }^{27}$ a conduit une génération entière d'historiens à poser la question des mémoires de la Révolution; et celle de François Hartog, lui-même inspiré par Reinhart Koselleck, et dont le concept de régime d'historicité est ici structurant. Pierre Serna revient sur les tentatives de mémorialiser la Révolution sous la Restauration et au début de la monarchie de Juillet en constatant qu'aucune d'entre elles ne parvient à imposer sa grille de lecture et de compréhension de l'évènement révolutionnaire. Pour lui, on touche ici à un problème intrinsèque à la mémoire de la Révolution. En effet, soit la mémoire de la Révolution est « une ossification de la rupture en une sédimentation qui la rend caduque et la termine ", soit « [elle] est un savoir-faire la Révolution subversif, et faire de la Révolution une mémoire, c'est provoquer la Révolution à son tour comme invention du futur par le détour de la mémoire ${ }^{28} »$. On a donc affaire ici à deux intentions de mémoire qui sont complètement irréconciliables, dans la mesure où leurs buts - conclure ou au contraire reprendre la Révolution - sont antagonistes. La deuxième partie de la contribution est dédiée à l'étude de la commande, de l'exposition et de la réception de deux peintures par la jeune monarchie de Juillet. Deux toiles sont en effet réalisées pour entourer la peinture présentant Louis-Philippe prêtant serment à la Charte, exposée dans la Chambre des députés. Chacune représente la loi s'opposant à une forme de tyrannie : dans la première, on voit Mirabeau s'élevant contre DreuxBrézé ; dans l'autre, Boissy d'Anglas, président de la Convention, doit saluer la tête du député Féraud. L'absolutisme royal et la foule révolutionnaire sont donc présentés comme l'avers et le revers de la même tyrannie. Mais la deuxième peinture, très critiquée par l'opposition lors de ses présentations à Nantes ou à Lyon, est progressivement réinvestie par celle-ci d'un sens très contemporain. Cela signe l'échec de cette tentative de l'extrême centre de refroidir la mémoire de la Révolution, celle-ci redevenant l'horizon d'une opposition qui s'identifie à la colère du peuple représentée sur le tableau. Pour Pierre Serna, c'est le signe que la seule mémoire de la Révolution ne pourra jamais conduire qu'à la répétition de la Révolution et de la Contre-révolution ; au contraire, c'est l'histoire qui peut s'arracher au régime de la mémoire pour participer aussi à la fondation de la République.

Dans « Des mots pour dire Robespierre », Hervé Leuwers étudie un travail inachevé, à la frontière de l'histoire et de la mémoire : l'ensemble des notes prises en exil par MarcAntoine Baudot, ancien conventionnel régicide, en vue d'écrire ses mémoires. Dans cette source originale, l'auteur laisse voir une ambition multiple: comprendre les évènements dont il a été un des acteurs; revendiquer l'héritage de l'an II et en conserver une trace; et attaquer Robespierre. Hervé Leuwers donne ainsi à voir un 
phénomène assez rarement capturé sur le vif - le mémorialiste au travail - alors que ce témoin de la Révolution française, qui paye le prix de ses engagements de jeunesse plus de trente plus tard, tente de se faire historien. Il expose notamment la génétique complexe de cet ensemble de notes entre souvenirs personnels, analyses sur l'époque et copies d'autres documents de l'époque révolutionnaire. D'autre part, ces notes donnent à voir l'effort que fait un opposant à Robespierre pour en formuler un " portrait vrai et nuancé29 ", loin des accusations fantaisistes que ses détracteurs (de son vivant et surtout après sa mort) ont lancées contre lui. Il repousse ainsi les nombreuses accusations de perversion sexuelle ou de débauche et il refuse également de prendre au sérieux l'hypothèse d'un Robespierre traître à la partie. Mais, dans cette critique révolutionnaire de l'Incorruptible, il voudrait par contre fixer l'image d'un Robespierre ambitieux ayant aspiré à la dictature. Pour Hervé Leuwers, ce rare brouillon d'un mémorialiste au travail permet de saisir un moment important de la construction mémorielle de Robespierre, autant qu'il nous montre un conventionnel régicide qui, trente ans après, « cherche encore à comprendre $\mathrm{e}^{30}$ ".

Avec «Les ego-documents et la mémoire des villes atlantiques ", Éric Saunier analyse trois ego-documents, écrits entre 1779 et 1830, au prisme de l'histoire urbaine : il s'agit de rendre compte de la formation de la mémoire des habitants du Havre en utilisant des textes qui se situent hors du champ des écrits destinés à raconter l'histoire de la ville. Leurs trois auteurs sont d'autant plus intéressants qu'ils se situent tous en dehors du milieu de l'élite négociante trop souvent étudié par les historiens pour rendre compte de la mentalité des villes de la bourgeoisie portuaire. Bien que les trois textes fussent écrits par des auteurs aux opinions politiques différentes - un monarchiste nostalgique, un fonctionnaire de l'Empire et un bonapartiste -, ils témoignent de soucis communs. Ceux-ci sont de deux ordres : d'abord, ils écrivent tous dans une perspective régionale, que cela soit pour rappeler l'identité catholique ou l'anglophobie d'une ville marquée par le conflit franco-anglais; ensuite, les trois textes témoignent d'une sensibilité atlantique en construction, que cela se fasse par un sentiment de communauté culturelle et historique avec les autres ports français, comme Nantes, ou à travers les références nombreuses à un monde colonial bouleversé par la Révolution. C'est sur ce sentiment atlantique de la communauté havraise qu'Éric Saunier conclut en soulignant qu'il est trop souvent absent des chroniques et annales historiques.

Dans sa contribution, "Le contrôle de la mémoire dans la France de Napoléon I ${ }^{\mathrm{er}}$ ", Veronica Granata s'intéresse à Delisle de Sales, un auteur éclectique qui, des dernières années de la monarchie à l'Empire, a eu maille à partir avec la censure. En retraçant l'itinéraire de ce "philosophe" en guerre contre tous les pouvoirs qu'il identifiait comme despotiques, l'historienne rend compte des évolutions de la censure depuis sa première incarcération en 1777 jusqu'à 1810, lorsque sa contribution aux Éléments d'histoire générale, commencée par l'abbé Millot et consacrée à cette Révolution que Delisle de Sales attaquait violemment, fut censurée D'une part, l'itinéraire tortueux de cet auteur sans cesse déçu par les régimes qui se succèdent à partir de 1789 permet de mesurer les hésitations du pouvoir impérial à mettre en place une censure préventive et les adaptations de celle-ci aux équilibres politiques de l'Empire, entre nostalgiques de la République et monarchistes ralliés. Mais cette contribution est aussi l'occasion de rendre compte des ambiguïtés de l'Empire, et de Napoléon lui-même, impliqué directement dans la censure des histoires de la Révolution que publie Delisle de Sales afin de fabriquer une mémoire impériale des années révolutionnaires. Les années 1810 et 1811 marquent alors un tournant, quand le régime décide de censurer 
systématiquement les histoires de la Révolution qui se publient, faisant silence sur « un passé qu'il perçoit désormais comme inutile pour justifier sa propre existence ${ }^{31} »$.

À travers la figure de La Tour d'Auvergne, «le quatrième mousquetaire de la Révolution ", Alain Le Bloas écrit aussi sur les processus de construction mémorielle, de la période consulaire à la monarchie de Juillet. En effet, «le premier grenadier de la Révolution ", comme le surnomma Napoléon, mort en 1800 au combat d'Oberhausen, fut l'objet d'une héroïsation par étapes qui permet de saisir les ressorts de la fabrique d'un héros, d'abord sous le Consulat puis, après une longue éclipse, sous la monarchie de Juillet. Alain Le Bloas s'intéresse autant aux modes de diffusion de la figure de La Tour d'Auvergne - par le portrait, la gravure ou la statuaire - qu'aux différentes facettes d'un héros approprié par plusieurs régimes et à différentes échelles. La Tour d'Auvergne est ainsi représenté comme un héros des guerres de la Révolution à la période consulaire et, plus tard, sous la monarchie de Juillet, comme une figure reprise par la gauche républicaine mais aussi par la droite légitimiste, témoignant de l'évolution de cette dernière vers le nationalisme et la défense des valeurs militaires. Alain Le Bloas souligne néanmoins que la plasticité du personnage - à la fois guerrier et philosophe, homme des Lumières revendiquant un nom aristocratique sous la Révolution - est un revers de sa complexité. C'est pour cela qu'il est difficilement approprié par le Consulat, l'Empire et, plus tard, la monarchie restaurée. En revanche, il devient un héros officiel à l'échelle régionale, en Bretagne, où il est célébré dans les années 1830 comme le plus grand héros de Carhaix, sa ville natale. C'est ainsiqu'il peut devenir un héros orléaniste, faisant de la monarchie de Juillet le premier régime à consacrer la mémoire de cette figure des guerres de la Révolution.

Après La Tour d'Auvergne, Bayard breton, les deux dernières contributions de cette partie consacrée aux mémoires individuelles traitent également des mémoires bretonnes de la Révolution. Dans la première, "Les Révoltés de Fouesnant ramenés à Quimper par la Garde nationale en 1792, Jules Girardet, 1886-1887: un tableau emblématique de la représentation de la Révolution en Bretagne ", Sophie Kervran montre comment, à l'orée du centenaire de la Révolution, un tableau présentant la répression des premières résistances paysannes à la Nation participe de la construction d'un héritage breton de la Révolution. Cette peinture d'histoire, un genre très en vogue dans les années 1880, s'inscrit dans un contexte dans lequel la Bretagne est peinte comme une terre chouanne jusqu'à la saturation. Cette peinture est résolument inspirée par les Chouans de Balzac et Quatrevingt-treize de Victor Hugo et met en avant une vision folklorique du paysan breton, sans qu'il soit possible d'affirmer une orientation républicaine ou, au contraire, contre-révolutionnaire de Jules Girardet. Il s'agit avant tout de consolider le souvenir de la Révolution. La peinture suscite pourtant un fort engouement en Bretagne, Sophie Kervran rappelant que les nombreuses reproductions de l'œuvre participent à en faire l'une des images de la Révolution dans la religion, sans que le public ne se souvienne plus de l'épisode qu'il représente ou de sa potentielle charge contre-révolutionnaire. On voit ici comment, d'erreur historique en erreur historique, les mémoires de la Révolution s'affranchissent de l'évènement originel pour se charger des significations successives qui leur sont données.

Sébastien Carney examine un autre aspect des mémoires bretonnes de la Révolution, ou plutôt de la contre-révolution. Dans « Une mémoire de 1789 dans le mouvement breton. Yann Fouéré, un contre-révolutionnaire en Révolution nationale", il retrace le parcours 
intellectuel d'un militant du mouvement breton qui voit dans l'occupation et la Révolution nationale une possibilité d'affirmer, sinon l'indépendance, au moins l'autonomie de la Bretagne. C'est pendant cette période que ce non-conformiste né en 1910 dans une famille républicaine va remplacer le Moyen-Âge par la Révolution dans l'écriture d'une histoire instrumentalisée par le mouvement breton. Sébastien Carney restitue les contours d'une pensée contre-révolutionnaire très marquée par les historiens bretonistes du XIX ${ }^{e}$ siècle, mais aussi par les courants décentralisateurs et provincialistes qui s'exprimèrent dans l'Action française. Il n'arrive que progressivement à cet héritage : c'est d'abord une Histoire de la Bretagne manuscrite de son oncle, dans laquelle s'expriment des tendances décentralisatrices; au cours des années 1920 et 1930, il est fasciné par la figure de Mussolini tout en critiquant de plus en plus un jacobinisme qui, pour lui, a trahi la Révolution ; la Seconde Guerre mondiale le voit définitivement basculer vers la Contre-révolution, puisqu'il défend la constitution d'un province bretonne réunissant les cinq départements historiques. Déçu par la Révolution nationale, qu'il dénonce comme une entreprise strictement réactionnaire, il prône de plus en plus la Révolution contre l'État, mais sur des fondements hostiles à la doctrine émancipatrice de la Révolution. Sébastien Carney conclue cette fascinante biographie en suggérant que, plus qu'un contrerévolutionnaire, Yann Fouéré serait un anti-révolutionnaire, c'est-à-dire un révolutionnaire déçu par la Révolution - selon la définition de Claude Mazauric - jeté dans la Révolution nationale.

Avec cette trajectoire individuelle, qui fait écho à celle d'autres militants du mouvement breton pendant la Seconde Guerre mondiale, se conclut la première partie de ces histoires de la mémoire de la Révolution. Celles-ci seront ensuite envisagées à l'échelle collective, que ce soit dans la culture, dans les pratiques ou dans des espaces définis. À chaque fois, il s'agit de démêler les fils de mémoires singulières qui, côte à côte et parfois antagonistes, esquissent les ressorts des mémoires françaises de la Révolution.

Philippe Bourdin s'intéresse au « Répertoire théâtral (1789-1795)» comme « espace de construction d'une mémoire immédiate ». De fait, en partant du constat que l'histoire de la Révolution s'est écrite dès 1789 , il rappelle que les arts, et notamment les arts du spectacle, ont été l'un des principaux vecteurs d'un récit en construction. Il rappelle d'abord les très nombreux travaux et initiatives collectives qui sont menés depuis une trentaine d'années et qui témoignent d'un intérêt nouveau pour les arts du spectacle. À la suite de cette impressionnante production historiographique, il développe trois axes de réflexion autour du théâtre comme espace de construction mémorielle : l'utilisation de la tragédie comme propédeutique révolutionnaire et dénonciation de la tyrannie; le récit théâtralisé des évènements contemporains, qui représente une part minoritaire mais importante du total des pièces jouées ; la pure fabrique mémorielle, qui passe par la commémoration des héros révolutionnaires, comme Marat ou l'anticipation d'utopies républicaines. Philippe Bourdin conclut néanmoins en montrant que les nombreux pièges et difficultés soulevés par un outil de propagande malaisé sont à la mesure des espoirs qu'ils suscitent chez certains révolutionnaires.

Cette contribution artistique fait écho à celles de Christian Amalvi et de Pascal Dupuy. La première, «Villes sculptées au XIX siècle. Les représentations de la Révolution française dans l'espace public, de 1830 à 1899 », se situe dans le sillon historiographique tracé par Maurice Agulhon. Christian Amalvi rappelle d'abord la multiplication des statues et 
plaques qui, dans la décennie 1880-1889, viennent commémorer la Révolution dans l'espace public alors que la République a finalement été conquise par les Républicains. Rappeler l'existence de ce patrimoine est d'autant plus crucial que le régime de Vichy, pour des raisons politiques, ainsi que la transformation radicale des centres villes français à partir des années 1950, ont provoqué sa disparition partielle. L'historien suggère néanmoins d'étudier cette statuaire mémorielle sur un temps plus long, en allant de la Révolution de 1830 jusqu'à 1899. Pour lui, la statuaire de la monarchie de Juillet dans ses années libérales, dont la trace la plus importante est la colonne de la liberté place de la Bastille qui unit le souvenir des mois de juillet 1789 et 1830, fut étendue et développée par la $\mathrm{III}^{\mathrm{e}}$ République. La $\mathrm{II}^{\mathrm{e}}$ République, au contraire, vit sa politique mémorielle captée et modifiée en profondeur par le Second Empire, comme l'illustrent les modifications apportées au projet de statue du maréchal Ney. La démission de Mac Mahon en 1879 ouvre une nouvelle période faste pour la statuaire révolutionnaire et, pendant vingt ans, la $\mathrm{III}^{\mathrm{e}}$ République s'emploie à fixer par la pierre et le bronze une narration conciliatrice et unificatrice de la Révolution française inscrit dans un long récit national d'une France hostile à la tyrannie (ce qui explique l'érection de statues d'Étienne Marcel et de l'amiral de Coligny). En conclusion, Christian Amalvi suggère que ce fonds mémoriel est parfois réactivé à l'aune des crises politiques contemporaines, comme lors des manifestations du 11 janvier 2015 qui, à Paris, ont vu des millions de personnes converger vers les statues des places de la Bastille et de la Nation.

Pascal Dupuy propose un texte sur «La mémoire de la Révolution française dans la musique populaire aux $\mathrm{xx}^{\mathrm{e}}$ et $\mathrm{xxI}^{\mathrm{e}}$ siècles. La révolution enchantée?». Comme la contribution de Philippe Bourdin, elle est marquée par le développement récent des recherches sur l'industrie musicale actuelle. L'étude se développe en trois axes qui articulent les principales réinterprétations musicales de la Révolution et de l'Empire depuis les années 1960. D’abord, Pascal Dupuy brosse un large tableau des références qui, depuis un demi-siècle, ont été faites à la Révolution dans la musique populaire. Si la figure de Napoléon semble être la référence principale jusque dans les années 1970, Pascal Dupuy montre que des musiques franchement contestataires, comme le rock québécois ou le punk, savent s'emparer de la Révolution. Le deuxième axe étudie les reprises de la Marseillaise et les réceptions qu'elles suscitent : le cas de Serge Gainsbourg est évidemment central, mais on se rend compte que la charge mémorielle de l'hymne national en fait un morceau souvent repris pour sa force symbolique. Enfin, Pascal Dupuy s'intéresse à la manière dont la mémoire de la Révolution est alimentée par la production musicale. L'enregistrement, depuis les années 1950, de chants de l'époque révolutionnaire a participé à la préservation d'un important patrimoine musical et à sa reconnaissance par un plus vaste public. Par ailleurs, depuis La Révolution française, premier opéra rock produit en France en 1973, on se rend compte que les adaptations scéniques suivantes de la décennie révolutionnaire, jusqu'aux Amants de la Bastille (2014), témoignent de la moindre charge révolutionnaire pour le grand public de la Révolution. Cette affirmation est néanmoins nuancée par la persistance de la Révolution comme objet d'appropriation musicale, aussi bien en France que dans le monde anglo-saxon.

Laurent Brassart signe quant à lui l'une des deux études régionales de cet ensemble : "Percer un brouillard mémoriel. Retrouver la trace de la Révolution française dans le NordPas-de-Calais (1789-1989) ». Il part d'un paradoxe: alors que le poids mémoriel de la Révolution dans le Nord est au cœur de deux monuments littéraires, Archives du Nord de 
Marguerite Yourcenar et Germinal de Zola, la Révolution est largement effacée du Nord. Pour comprendre les ressorts de cette contradiction, Laurent Brassart présente une enquête fine et précise, en plusieurs étapes. Il montre tout d'abord que la Révolution a laissé peu de monuments - deux seulement ont survécu jusqu'en 1989 - et que, dans la masse des mémoires publiés par des contemporains de la Révolution, le Nord fait partie des régions peu représentées. De plus, ces textes défendent rarement la Révolution, puisque la majorité est contre-révolutionnaire, les autres adoptant un ton neutre ou modéré. Les monuments érigés après la Révolution pour la commémorer témoignent également de fortes disparités spatiales entre un Nord révolutionnaire et un Pas-deCalais plus neutre. C'est le résultat de la polarisation politique d'une région partagée entre des territoires acquis à la République (la métropole lilloise se taillant la part du lion) et des territoires encore sous emprise de l'Église. Le centenaire et le centcinquantenaire de la Révolution ne modifient pas ces équilibres qui se pérennisent dans l'espace et dans les mémoires. Malgré tout, si la mémoire de la Révolution demeure dans d'anciens bastions socialistes, les zones rurales ne sont pas devenues des conservatoires de la mémoire contre-révolutionnaire. Le fait que le Nord et le Pas-deCalais aient été de fortes zones d'immigration a aussi contribué à infléchir la stratégie mémorielle des partis de gauche, qui ont préféré concentrer leur discours sur les souffrances immédiates du mouvement ouvrier que sur un passé révolutionnaire trop lointain. Cette contribution dense rend compte avec beaucoup de finesse des modalités de la conservation ou au contraire de l'effacement de la Révolution dans un territoire donné. On se rend compte combien cette mémoire est dépendante d'une multiplicité de facteurs, des équilibres politiques et religieux d'une région aux questions sociales soulevées par une région métamorphosée par la Révolution industrielle.

Dans l'autre contribution régionale, « Entretenir la mémoire contre-révolutionnaire au XIX ${ }^{e}$ siècle. Lieux et pratiques dans la France du Sud-Est » Bruno Dumons prend le cas ce territoire pour étudier un phénomène continental, la persistance de la mémoire de la Contre-révolution, dans le sillage de ses travaux sur la Contre-révolution et le catholicisme en Europe à l'époque contemporaine. Dans un grand Sud-Est, commençant à Lyon et englobant la Provence blanche - véritable pendant de l'Hérault blanc étudié par Philippe Secondy ${ }^{32}$-, Bruno Dumons montre que ces territoires sont loin d'être isolés et que, au contraire, ils sont mis en réseau par des réseaux de sociabilité et des circuits mémoriels qui font perdurer une profonde culture contre-révolutionnaire, aussi bien dans les familles aristocratiques que bourgeoises. Cette contribution ne prétend pas à l'exhaustivité dans un champ aujourd'hui très actif (et dont Bruno Dumons est l'un des animateurs), l'historien cherchant plutôt à identifier et décrire quelques-uns des vecteurs de cette mémoire blanche : les pratiques généalogiques qui organisent le culte des ancêtres contre-révolutionnaires; l'éducation dans les collèges jésuites (à Fribourg en Suisse jusqu'en 1847 et ensuite à Avignon), qui jettent les bases d'une véritable " armée "blanche" en exil ${ }^{33}$ " qui se traduit notamment par l'expérience combattante des zouaves pontificaux; les banquets et pèlerinages auprès du comte de Chambord, dont les récits alimentent le souvenir et l'espérance de la Restauration. L'article démontre, s'il le fallait encore, la nécessité de continuer à étudier cet objet protéiforme et longtemps ignoré, la Contre-révolution et sa mémoire.

Enfin, trois contributions s'intéressent à des périodes de la mémoire de la Révolution. Dans « Les usages politiques de l'histoire et de la mémoire de la Révolution française de 1880 à 1914 ", Jean El Gammal donne un aperçu synthétique des permanences et des inflexions de l'histoire et de la mémoire de la Révolution à l'époque de la « République 
triomphante ». Ce texte, nourri par les travaux de Pascal Ory et, plus récemment, de Jean-Numa Ducange, s'intéresse d'abord aux conflits mémoriels et historiographiques que suscitent la Révolution alors que la République s'installe. Le format des célébrations, nationales ou privées, et des contre-célébrations, largement organisées autour de l'Église, est également évoqué. Finalement, Jean El Gammal souligne que le centenaire de la Révolution et la période 1898-1900 sont deux moments qui voient l'historiographie et la mémoire de l'évènement s'infléchir. Cet article, qui se présente comme une synthèse et un état de la recherche, ouvre des pistes sur la question de la mémoire dans ces années d'enracinement de la République, pourtant marquées par de profondes crises politiques.

De la mémoire vive à l'histoire vivante, Danielle Tartarowski, dans la « Révolution du Front populaire ", propose une étude très complète de la manière dont la mémoire et l'histoire de la Révolution sont mobilisées et renouvelées par la période qui, de 1934 à 1939, voit émerger la fondation, l'élection et la disparition du Front populaire. En reprenant la distinction opérée par Marie-Claire Lavabre entre "mémoire historique ", transmise par l'école ou des organisations, et la "mémoire vive», mobilisable spontanément en dehors du cadre commémoratif, Danielle Tartarowski montre comment, des manifestations des 6 et 12 février 1934 jusqu'aux commémorations, le plus souvent discrètes, du cent-cinquantenaire, la Révolution est mobilisée par les protagonistes de ce moment de renouvellement politique. La nécessité de faire front contre une menace identifiée comme fasciste pousse notamment le PCF et la SFIO à s'emparer de la Révolution, en plus de leurs nombreuses références au mouvement ouvrier, pour réactiver en 1936 l'opposition entre Révolution et Contre-révolution, identifiant les fascistes aux « coblençards » de 1792. La victoire du printemps amplifie ce phénomène de renouvellement mémoriel tout en suscitant de nouvelles recherches historiques, un exemple intéressant de l'influence des mémoires politiques sur le champ universitaire. Enfin, les difficultés du Front populaire et les tensions internationales croissantes contribuent au reflux de ce phénomène, sans qu'il n'ait laissé aucune trace : Danielle Tartarowski montre comment le Parti communiste s'est pleinement emparé de la mémoire de la Révolution, jusqu'à la toponymie d'une station de métro à Montreuil. Ce texte articule très finement les nombreux objets - histoire, mémoire, commémoration, transmission, inflexion - qui travaillent l'ensemble de l'ouvrage et rend compte de l'immense activité déployée par le Front populaire dans le domaine des représentations historiques.

La contribution de Laurent Douzou, «L'histoire de la Révolution française dans la mémoire et les pratiques de la Résistance ", est une riche étude mémorielle qui a l'intérêt de s'inscrire dans le temps long du travail d'un chercheur, puisque ce texte fait référence à l'une de ses premières communications, lorsqu'il était encore doctorant sous la direction de Maurice Agulhon. Ici, il s'emploie à nuancer une impression sans doute largement partagée : le lien entre mémoire de la Révolution et Résistance serait simple et évident, comme en témoignent les nombreuses références que la seconde fit à la décennie révolutionnaire dans sa lutte contre l'occupant et un régime de Vichy hostile aux symboles et à l'héritage révolutionnaires de la République. En réalité, la nature des liens entre la Révolution et la Résistance fut complexe et s'établit selon différentes modalités. L'histoire de la Révolution fut bien sûr un vivier de références mobilisées dans la presse résistante: encore plus que la réalité politique de la Révolution, ce sont les guerres de libération du territoire menées par celle-ci qui séduisaient la Résistance intérieure. Mais la nouveauté du phénomène résistant dans 
l'histoire, revendiquée par ses acteurs, rendait parfois malaisée la référence au passé. Cette singularité de l'expérience résistante, lutte clandestine contre l'ennemi là où la Révolution avait été une "lutte à ciel ouvert ${ }^{34}$ ", explique pour Laurent Douzou pourquoi ce sont les valeurs révolutionnaires et républicaines qui ont été mobilisées plutôt que l'histoire de la Révolution à proprement parler. Son article se termine par une fine réflexion sur la différence entre une Résistance " milieu de mémoire », fragile et dépendante de la mémoire de ses protagonistes, et une Révolution «lieu de mémoire », à la puissance évocatoire installée.

À raison, Anne de Mathan, qui a su impulser une vraie direction à cet ouvrage, rappelle dans sa conclusion que la diversité des contributions et le vaste spectre des thèmes et des questions soulevés sont une richesse et non pas un handicap, d'autant plus qu'elle trouve une unité souterraine à ces textes en montrant que, pour tous ces auteurs, le véritable intérêt de la mémoire réside beaucoup plus dans ce qu'elle dit des individus ou des groupes qui la transmettent ou la reçoivent que dans la nature des souvenirs qu'elle porte : «c'est finalement l'histoire, la sociologie, l'historiographie ».

qu'il en soit, un livre d'histoire - de sciences sociales même - aussi ambitieux que celui-ci établit la richesse et les difficultés qu'il y a à entreprendre une telle réflexion kaléidoscopique. S'il est tout à fait profitable de lire les Mémoires de la Révolution française de manière linéaire, et tout particulièrement la première partie, dans laquelle on retrouve une progression théorique bien identifiable, le foisonnement des deux parties suivantes invite aussi à une lecture plus désordonnée qui permet d'avancer par grappes de thématiques qui articulent la mémoire à d'autre problèmes: approches régionales, histoire de l'art, figures de la Révolution, etc.

41 Trente-cinq ans après la publication du premier volume des Lieux de mémoire, ouvrage fondateur dont nombre de contributions discutent pour mieux s'en démarquer, Mémoires de la Révolution française se présente à la fois comme un aboutissement de la recherche de nombre des contributeurs, et comme le point de départ d'une infinité de chantiers. C'est l'une des grandes qualités de cet ouvrage que de susciter dès son introduction la curiosité du lecteur, qui restera sans cesse stimulée par des contributions le plus souvent riches et denses et qui ouvrent de nombreuses pistes de réflexions. À n'en pas douter, ce livre sera à l'origine d'articles et d'ouvrages qui continueront de cartographier les multiples formes que la mémoire de la Révolution a prises dans l'espace et dans le temps depuis 1789.

À la fin des Mémoires de la Révolution française, on aimerait notamment poursuivre le travail entamé dans deux directions. Si l'ouvrage, et notamment la deuxième partie, fait un vrai effort pour ne pas se limiter au strict cadre français, la majorité des contributions s'inscrivent dans un cadre national, français ou étranger. Ce cadre n'est remis en question que par les contributions qui interrogent les mémoires régionales de la Révolution. D’autres échelles auraient pu être mobilisées : à l'échelle régionale, en prenant par exemple des cadres transfrontaliers ; à l'échelle continentale, en évoquant la possibilité de mémoires européennes; dans des espaces linguistiques précis, comme la contribution d'Annie Jourdan, qui exposent les prémisses de la fabrication de mémoires anglophones de la Révolution. Quand bien même la Révolution est

La Révolution française, 17 | 2020 
inséparable de la catégorie politique de la nation, ces variations d'échelles permettraient peut-être de faire émerger d'autres mémoires interstitielles en articulant des mémoires qui s'expriment dans des cadres spatiaux différentiés.

La Contre-révolution est l'objet central de quatre des contributions - celles de JeanClément Martin, Paul Chopelin, Sébastien Carney et Bruno Dumons -, faisant justement toutes remarquer qu'elle est inséparable de l'étude de la Révolution, qui ne peut être comprise qu'imparfaitement sans elle. Bien que la lecture de ces quatre textes (et des quelques autres qui lient Révolution et Contre-révolution) soit absolument passionnante, leur rareté pourrait donner l'impression d'un éparpillement ou d'une discontinuité des pratiques mémorielles contre-révolutionnaires en totale contradiction avec la force de frappe mémorielle d'institutions (comme l'Église), de réseaux (familiaux et institutionnels) et de territoires qui ont assuré la persistance du souvenir des luttes de la Contre-révolution et de son corrélat, la haine renouvelée de la Révolution et de ses idées. Cette mémoire devient d'autant plus importante que, en France au moins, la consolidation de la République prive ces adversaires de la Révolution de nombreux relais institutionnels. Leur survie idéologique ne peut alors passer que par une survie mémorielle.

Si ces Mémoires de la Révolution française restent un livre nécessaire pour les spécialistes de la période, la volonté d'y mettre en avant de nombreuses facettes de la relation complexe qui noue l'historien à la question de la mémoire l'ouvre sans aucun doute à un public plus large parmi tous ceux qui s'interrogent sur les modalités variées de notre relation au temps passé.

\section{NOTES}

1. Anne de mathan (dir.), Mémoires de la Révolution française, Rennes, Presses universitaires de Rennes, 2019, p.7.

2. Anne DE MATHAn, Mémoires de Terreur: l'an II à Bordeaux, Pessac, Presses Universitaires de Bordeaux, 2002, 232 p. Histoires de Terreur. Les mémoires de François-Armand Cholet et Honoré Riouffe, Paris, Honoré Champion, 2014, 368 p.

3. Anne De mathan (dir.), Mémoires de la Révolution française, op. cit., p. 9.

4. Anne DE MATHAN (dir.), ibid., p. 15.

5. Anne DE MATHAN (dir.), ibid.

6. Pierre NORA (dir.), Les lieux de mémoire (3 vol.), Paris, Gallimard, 1984-1994.

7. Anne DE MATHAN (dir.), Mémoires de la Révolution française, op. cit., p. 21.

8. Johann MICHEL, « Travail de mémoire, devoir de mémoire, devoir d'histoire, travail de l'histoire ", in Anne de Mathan (dir.), Mémoires de la Révolution française, op. cit., p. 63

9. Philippe JOUTARD, « Histoire et mémoire. Deux approches du passé souvent en conflit mais qui doivent s'articuler », in Anne de Mathan (dir.), Mémoires de la Révolution française, op. cit., p. 65.

10. Philippe JOUTARD, « Histoire et mémoire. Deux approches du passé souvent en conflit mais qui doivent s'articuler », in Anne de Mathan (dir.), Mémoires de la Révolution française, op. cit., p. 65.

11. Philippe JOUTARD, ibid., p. 67. 
12. Philippe JOUTARD, ibid., p. 68.

13. André SIEGFRIED, Tableau politique de la France de l'Ouest, Paris, Armand Colin, 1913, $536 \mathrm{p}$.

14. Notamment Jean-Clément MARTIN, La Vendée de la mémoire, 1800-1980, Paris, Éditions du Seuil, 1989,298 p.

15. Sylvie SAGNES, «De passeur à décrypteur de mémoire ... et retour », in Anne de Mathan (dir.), Mémoires de la Révolution française, op. cit., p. 91

16. Sylvie SAGNES, ibid.

17. Jean-Clément MARTIN, La Vendée et la Révolution : accepter la mémoire pour écrire l'histoire, Paris, Perrin, 2007, $283 \mathrm{p}$.

18. Jean-Clément MARTIN, "Quand l'histoire bute sur les mémoires, le cas de la Révolution française ", in Anne de Mathan (dir.), Mémoires de la Révolution française, op. cit., p. 94.

19. Jean-Clément MARTIN, ibid., p. 103

20. Annie JOURDAN, «Au-delà des frontières. Dialogues de sourds et miroirs déformants ", in Anne de Mathan (dir.), Mémoires de la Révolution française, op. cit., p. 111.

21. Ibid., p. 112.

22. Yannick Bosc, "Robespierre, la gauche et la fabrique négatif ", in Anne de Mathan (dir.), Mémoires de la Révolution française, op. cit., p. 123

23. Laurent TURCOT, Jean-Clément MARTIN, Au cour de la Révolution. Les leçons d'histoire d'un jeu vidéo, Paris, Vendémiaire, 2015, $144 \mathrm{p}$.

24. Jean-Numa DUCANGE, «Walter Markov : la "Grande Révolution française » vue de l'Est », in Anne de Mathan (dir.), Mémoires de la Révolution française, op. cit., p. 164.

25. Une notion largement développée par Gaël BRUSTIER dans Le Mai 68 conservateur. Que restera-t-il de la Manif pour tous?, Paris, Éditions du Cerf, 2015, 240 p.

26. Paul CHOPELIN, « Du Bicentenaire à la Manif pour tous. Les catholiques français et la mémoire de la "persécution révolutionnaire" (années 1980-années 2010) », in Anne de Mathan (dir.), Mémoires de la Révolution française, op. cit., p. 181.

27. Sergio LUZZATo, Mémoires de la Terreur: vieux Montagnards et jeunes républicains au XIX siècle, Lyon, Presses Universitaires de Lyon, 1991, 223 p.

28. Pierre SERNA, « Régimes de mémoires et mémoires de régimes. Une histoire impossible ?», in Anne de Mathan (dir.), Mémoires de la Révolution française, op. cit., p. 190.

29. Hervé LEUWERS, «Des mots pour dire Robespierre ", in Anne de Mathan (dir.), Mémoires de la Révolution française, op. cit., p. 198

30. Hervé LEUWERs, ibid., p. 205.

31. Veronica GRANATA, "Le contrôle de la mémoire dans la France de Napoléon Ier ", in Anne de Mathan (dir.), Mémoires de la Révolution française, op. cit., p. 230

32. Philippe SECONDY, La persistance du Midi blanc: l'Hérault, 1789-1962, Perpignan, Presses Universitaires de Perpignan, 2006, 393 p.

33. Bruno DUMONS, «Entretenir la mémoire contre-révolutionnaire au $\mathrm{xIX}^{\mathrm{e}}$ siècle. Lieux et pratiques dans la France du Sud-Est », in Anne de Mathan (dir.), Mémoires de la Révolution française, op. cit., p. 313.

34. Laurent Douzou, « L'histoire de la Révolution française dans la mémoire et les pratiques de la Résistance ", in Anne de Mathan (dir.), Mémoires de la Révolution française, op. cit., p. 350 


\section{AUTEUR}

\section{BAPTISTE ROGER-LACAN}

Doctorant en histoire

Centre Norbert Elias - IHRF-IHMC

Université d'Avignon et des Pays du Vaucluse - Université Paris 1 Panthéon-Sorbonne 